\title{
Autonomic management for convergent networks to support robustness of appliance technologies
}

\author{
Ahmad Kamal Ramli, Karim Djemame \\ School of Computing \\ University of Leeds \\ \{scakr,scskd\}@leeds.ac.uk
}

\begin{abstract}
Autonomic management within autonomic computing framework is considered as the future and viable solution for many appliances, either in software or hardware. Nevertheless, its current research application in computer networks is mainly visible in the intra domain space, and less attention is given to inter domain between one core network and another. This paper reviews some of the work on autonomic management and presents a framework that can be extended to a global and universal solution, such as fulfilling demand on bandwidth management, Quality of Service (QOS), and Service Level Agreements (SLA). The autonomic computing self-* features are considered to show the viability of the proposed framework.
\end{abstract}

\section{Keywords}

Autonomous and Adaptive Security, Information Assurance, Next generation Networks, Adaptive Architecture, Autonomic Management, Service Level Agreements, Bandwidth Management.

\section{INTRODUCTION}

Living in the modern technologies especially a combination of embedded system and hardware appliances leads to a huge demand for the Internet bandwidth, a global need that needs to be supported by Internet Service Providers (ISP). On the other side, the bandwidth utilization can either be on social activities, working or as complement of digital life, newer technologies such as grid computing, cloud computing, wireless, wired network, IPv6 till IPv8 etc. All those innovations are the key for the next generation networks, and support the Internet of things (IoT).

Permission to make digital or hard copies of part or all of this work for personal or classroom use is granted without fee provided that copies are not made or distributed for profit or commercial advantage and that copies bear this notice and the full citation on the first page. Copyrights for third-party components of this work must be honored. For all other uses, contact the Owner/Author.

Copyright is held by the owner/author(s).

SIN '14, Sep 09-11 2014, Glasgow, Scotland Uk

ACM 978-1-4503-3033-6/14/09.

http://dx.doi.org/10.1145/2659651.2660516
Traditionally, bandwidth allocation and scheduling techniques has been fixed as static conditions to ensure each of the applications or Internet resources are running on average speed, often helped by packet switching technology. However, static techniques are not efficient in allocating the left bandwidth for underutilized application and resources. Ideally, these underutilized resources could be made available to other applications/processes to ensure other actions could be performed and Quality of Service (QoS) delivered.

Translation of the resources demand, especially bandwidth, into reality is something not visible at this time in the terms of corporate environment and autonomic processes. Autonomic networking covers a large spectrum of themes with the introduction of "selfware" concept. The aim is to automate networking systems with intelligent capabilities to react to context changes with less intervention from user interaction. The development process will definitely need substantial research and certainly be evolutionary and gradual. With the ability to perform self-healing and self-reconfiguration many redundant and routine tasks can be done automatically while replacing or solving node failures in the network configuration, especially on the routing issues side.

The Internet Engineering Task Force (IETF) has produced a gap analysis of Autonomic Networking which covers the current application of autonomic technology in IP Address Management and DNS, Routing, Configuration of Default Routers, Hostname Lookup, User Authentication, and Accounting and Security [1]. There are three major situations in line with autonomic networking that are relevant but not observable in the analysis and which require human intervention, such as:

$\begin{array}{ll}\text { i. } & \text { Network Establishment } \\ \text { ii. } & \text { Network Maintenance and Management } \\ \text { iii. } & \text { Troubleshooting and Recovery }\end{array}$

In this paper, we propose a service-based adaptive framework based on autonomic management of network resources and appliances in the environment of service provider network and tiers. Several works have focused on small scale networks or known to be intra- domain network. However, the core issues on back bone enterprise networks are still to be researched. One of the difficulties is due to less ability to access the infrastructures that run identically like the real network architecture within Internet Service Provider level.

A substantial research element is the self-service features to ensure satisfactory bandwidth management within the providers and subscribers. To justify the process legally, it will be bound 
with Service Level Agreement, and Key Performance Indicators (KPI) will be the indicators for end user satisfaction.

Another important point of this paper is to identify the ability of autonomic management in handling multiple routing technologies in the network environment between one core router and another till the last edge or the exchange level. The outcome will then benefit the extended research on global bandwidth management and connectivity from one component to another connected within the network layers.

This paper is organized as follows. Background and related work is presented in section II. In Section III, we propose an architecture to enhance the productivity of a global network with the adaption of autonomic networking. The conclusion and immediate plan for the implementation of this research are explained in section IV.

\section{BACKGROUND}

The proposed autonomic computing by IBM [4] has the ability to manage the framework and free system administrators from routine tasks in a networking environment. Self-management embraces four elements;

\section{A. Self-Configuration}

In conventional computing, this is done by corporate data centres that have multiple vendors and platforms. Installing, configuring, and integrating systems is time consuming and error prone. However, in autonomic computing the automated configuration of components and systems follows high level policies. The rest of system components adjust automatically and seamlessly.

\section{B. Self-Optimization}

This feature ensures components and systems continually seek opportunities to improve their own performance and efficiency. This is difficult in current computing where systems have hundreds of manually set, nonlinear tuning parameters and their numbers increases with each release.

\section{Self-Healing}

Having this opportunity, autonomic computing is able to automatically detect, diagnose and repair localized software and hardware problems. This is so problematic in conventional approaches due to problem determination in large, complex systems that can take a team of programmer's weeks.

\section{Self-Protection}

In current practise, detection of and recovery from attacks and cascading failures is manual. Placing this feature will ensure the system automatically defends itself against malicious attacks or cascading failures. It uses early warnings to anticipate and prevent system wide failures.

Schroeder [2] introduced Autonet to forecast the possibilities of handling huge high speed data transaction in next generation approach. However J.M. Garcia and J. Duato [3] investigated the dynamic reconfiguration and focused on the transparent processing between one network node and another. Novelty of that research was to ensure the flow of data runs smoothly and does not affect applications within the network. In contrast, the approach is very much limited on one aspect of self -configuring and it is less inclusive model.

Autonomic Management as mentioned earlier became another element within autonomic networking that plays a major role in perfecting the whole four concepts of self-process. It is stated in the IBM blueprint paper v7 [8] that a number of factors crucially exist in the implementation namely:

1. Autonomic manager

2. Knowledge source

3. Touchpoint

4. Manual manager

5. Enterprise service bus

Figure 1 illustrates the concept of autonomic manager and the linkages with the adaptive process.

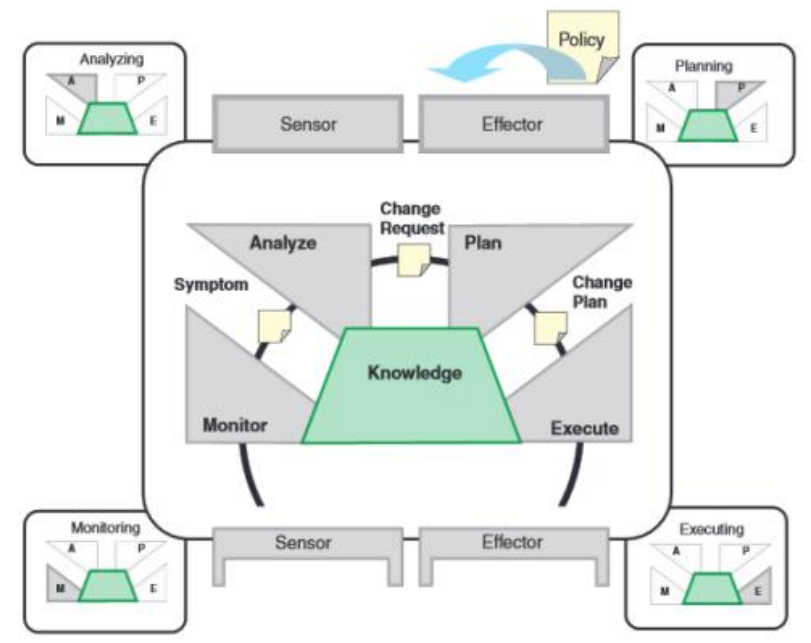

Figure 1: Functional details of the autonomic manager [8]

A number of researchers have done significant research on the autonomic management aspects. Knowledge base has been injected into research to justify the flexibility of taxonomy and semantics searching capabilities and can drive this autonomic management for better decision making [5]. The outcome was very positive, whereby self-management is able to understand the contents of predefined knowledge base. To extend the capabilities of autonomic management within the intra-domain capabilities, research made with Netserv [6] on a testbed environment is worth mentioning. This was followed by subsequent research [7] using virtualized networks to try to understand the failure nodes for better management thanks to a proposed algorithm. The outcome of this research stated that this proposed algorithm is able to recover the failure nodes and manage virtualized environment with less technical interference from human intervention.

Self-routing ability has been extensively reviewed by adopting the Extensible Open Router Platform with Great Plains Environment for Network Innovation [9]. Within this research, virtualized environments and wired topologies have been tested on routing issues. The end result confirmed that a virtualized network fully works and is able to route equally efficiently in a wired topology. 
Details on the actual test bed of that research is found in [10]. Great Plains Environment for Network Innovation (GENI) allows experimenters to obtain compute resources from locations around the United States, connect compute resources using Layer 2 networks in topologies best suited to their experiments, install custom software or even custom operating systems on these compute resources, and control how network switches in their experiment handle traffic flows. They can run their own Layer 3 and above protocols by installing protocol software in their compute resources and by providing flow controllers for their switches. The testbed is well suited for exploring networks at scale, thereby promoting innovations in network science, security, services and applications.

On the other side, Gamer and the team [11] have investigated security on the self-healing capacities over autonomic computing. According to their research it is proven that selfhealing algorithms are able to overcome malicious attacks ranging from virus, worms, DOS and DDOS. Having this result as the great start in security over autonomic management, further contributions can be further made to secure the network from unwanted outbreak and worsen network situation.

Lastly another two main contributions that are significant in the scope of this paper are self-management with the ability to support SLAs and manage bandwidth using autonomic management. The research by Tebbani and Aib [12] considering a language specifically defined known as GXLA, is able to apply self-management with SLA policies from end users and the service providers. In this work, VOIP was utilized to justify the connectivity using XML technologies during SLA exchanges between two parties.

In contrast, SLAs mainly focus on the satisfaction of one utilization which is VOIP and we know that elements within internet activities are more than that. On another issue this work is not being applied in the real time application or any critical application to ensure satisfaction from subscribers can be measured for better QoS. The framework of this exercise is also limited to small scale network domains and therefore further research should focus on large scale networks for more precise data and analysis.

On the perspective of bandwidth management allocation, the research in [13] focuses on the implementation of bandwidth management from one intra-domain to another. Autonomic management is the key of this exercise to ensure the availability of the bandwidth with sufficient justification between appliances. Although the result grants that autonomic management is able to control bandwidth allocation from one edge router to another end within the small network, a huge continuity of research should be examine the large scale of networks with various routing protocols. The Reason is to ensure the autonomic management with robust enhancement is able to manage all the differences in routing technologies.

Recently, Alcaraz [14] explored another method for supporting bandwidth reservation. Four main elements are highlighted: Primary Network (PN), Secondary Network (SN), Primary Users (PU), and lastly Secondary Users (SU ) as the perimeter that will be the input in the Markov Reward Model.
A bandwidth reservation scheme is proposed by which the PN keeps a set of adjacent channels free of PU transmissions. These reserved channels only accommodate PU traffic when all the non-reserved channels are used, and the SU only occupies the available channel within the reserved spectrum. In this theory, secondary users are not limited to persons; this can be extended to appliances, running algorithms such as Bayesian and multichannel access. The results show that, in non-congested PN with activity coming from Secondary Users, the interference reduction capability of Bandwidth Reservation increases the overall capacity of the PN compared to not using Bandwidth Reservation.

On the last note of this research, a framework should be present to ensure autonomic management has the capacity to govern the global network within one service provider to another and to ensure the adaptive environment is present to mitigate any uncertainty of network resources.

\section{PROPOSED RESEARCH}

The objective of next generation networks will be more robust than existing ones. Research is looking more into generic solutions of routing technology and eliminating proprietary routing technologies which are discovered individually by network providers and solution integrators.

In conjunction with that approach, at present we can identify a few prominent routing technologies in solving daily needs for internet activities, such as:

1. Open Shortest Path First (OSPF)

2. Border Gateway Protocol (BGP)

3. Integrated Services Digital Network (ISDN)

4. Multiprotocol Label Switching (MPLS)

5. Fiber Distributed Data Interface (FDDI)

6. Enhanced Interior Gateway Routing Protocol (EIGRP)

7. Routing Information Protocol (RIP).

In line with the proposed architecture, there are several research questions that need to be properly addressed and require a thorough investigation:

1. How does the existing (and different) routing topologies in the service provider can be bond into one solution?

2. How to ensure the bandwidth management can be tight back to back with SLAs between the end user and the network provider?

3. How to ensure a user gets the utmost utilization of bandwidth with his own application or packet preference?

4. How to ensure the availability of the network resources, such as bandwidth, routing nodes, and QOS issues can be managed properly using central autonomic management? 


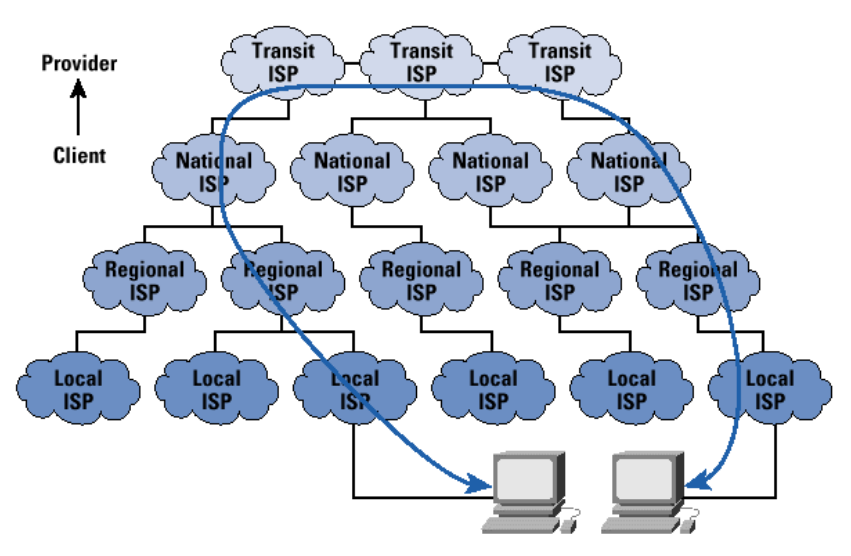

Figure 2. Internet Connectivity from multiple tiers and service providers [15]

Figure 2 shows the current internet with multiple tiers serving different needs for each player, such as businesses, consumers, retailers etc. By adopting a convergent approach, autonomic management is able to overcome tiers issues and over charging of bandwidth can be represented as follows;

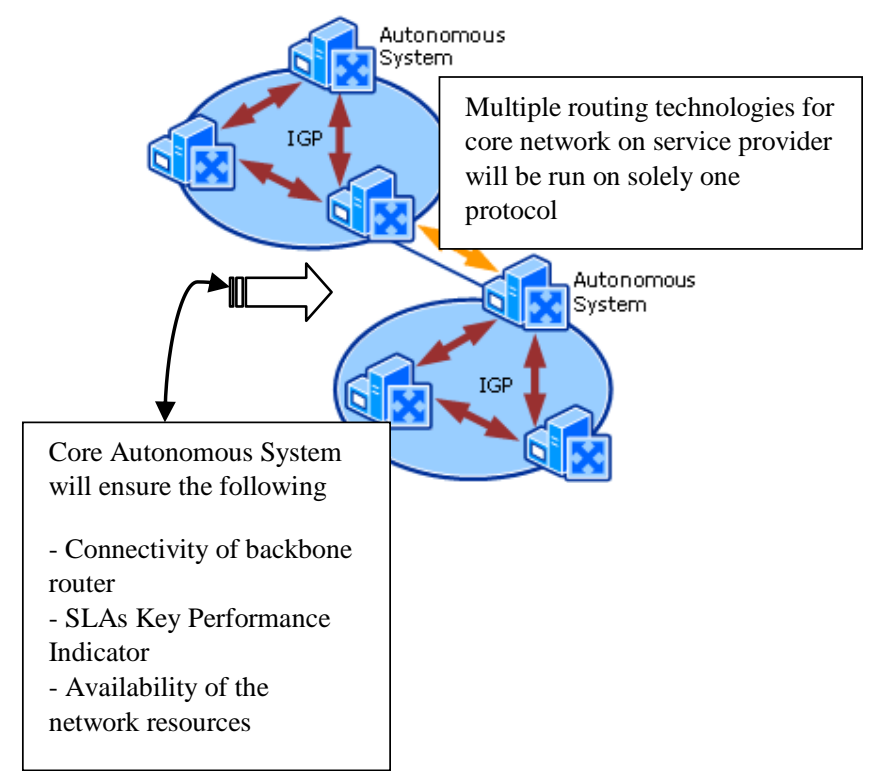

Figure 3. Global autonomous routing approach with existing local autonomous system

In a very conceptual execution, this implementation can be segregate into different policy based environments and managed by different authorization of autonomic managers. For example, on one hand a local network will be present with a local policy and works with a local autonomic manager, and on the other hand the global policy architecture is handled by a global autonomic manager. Logical concepts of this explanation are described in Figure 4.

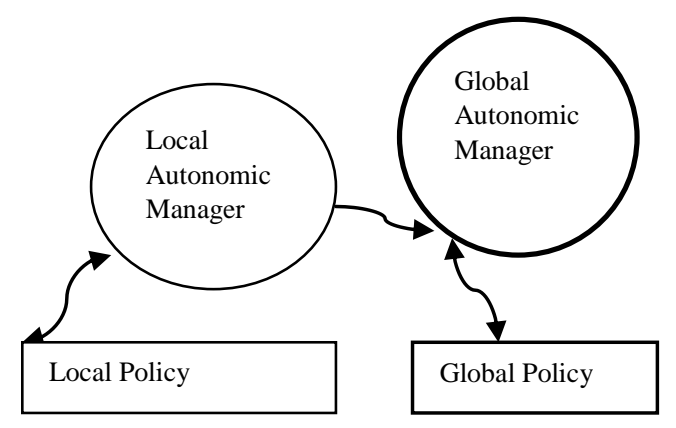

Figure 4. Logical concept of Policy Based Architecture between Local and Global Autonomic Manager

The stability of this conceptual framework is likely to be similar to existing solution for domain name server (DNS). In the DNS architecture the global architecture will communicate with local DNS server to understand what are the remaining virtual or sub directories that exist locally. For instance global DNS will understand global domain such as www.example.com, and within this domain the left sub domains will only be known if it as advertised by local DNS server. The possible sub directory will be site1.example.com, site2.example.com and etc.

By adopting such approach, the global autonomic manager will be established with the local autonomic manager using novel algorithm to manage the resources and policies that are presently connected. From there, necessary management process will be executed to ensure the stability of the network is preserved.

Having this approach as the solution, the next generation network will be ready for uncertainty of various bottleneck problems. This statement reflects the research questions addressed earlier in the following situation:

1. The growing of different and proprietary routing technologies will certainly create instability of network performance, services etc. Due to that a generic, robust and novel algorithm will ensure the connectivity is identical from one core network to another within the service provider network environment. Having this, the handshake and connectivity issues between routing protocols will be totally void.

2. With the introduction of enterprise autonomic management, the framework itself will be capable to manage various global parameters such as QoS, SLAs, User Profiling, Bandwidth Management etc.

3. The novelty of the solution will manage the autonomic framework which will be able to identify the current resources either on software or appliances to ensure the stability and connectivity between components under various conditions.

\section{CONCLUSION}

The future internet is something that people are looking forward to, and is under the responsibility of internet engineering task force (IETF), IEEE, RFC etc. With robust solutions presented 
daily, any mistake of the investment will turn one company's capital to be in red financial situation instantly.

It is clearly present in the literature that major research should be introduced to enhance the autonomic management abilities to be incorporated into enterprise solutions. There are two important milestones that need consideration.

The first one is to identify autonomic management in the capacity of the enterprise level in managing network resources in the inter-domain rather than the intra-domain. Having this will ensure the stability of network resources from the core router till the access level.

For the second one, since networks are becoming convergent, an adaptive framework should be present to efficiently control the bandwidth allocation and synchronize that with the users' behaviours while they are connected over the network. Having this control mechanism will be a preventive measure to mitigate social issues and usage based pricing of internet bandwidth.

\section{REFERENCES}

[1] M. Behringer, " Gap Analysis of Autonomic Networking ", Internet Engineering Task Force , Cisco Systems , April 2 2014 , Available online : http://tools.ietf.org/html/draft-irtfnmrg-an-gap-analysis-00

[2] Michael D. Schroeder , Andrew D. Birrell, Michael Burrows, Hal Murray and Roger M. Needham ," Autonet : A High Speed, Self-Configuring Local Area Network Using Point to Point Links “ , IEEE Journal On Selected Areas In Communication, vol 9 , no. 8, pp. 1318-1335, October 1991.

[3] J.M. Garcia and J. Duato , “An Algorithm for Dynamic Reconfiguration of a Multicomputer Network ", In Proceeding of the Third IEEE Symposiums on Parallel and Distributed Processing 1991, December 1991, pp. 848 855.

[4] Jeffery O. Kephart and David M. Chess, “ The Vision of Autonomic Computing “, Computer, Vol. 36, no. 1, pp. 4150, Jan. 2003.

[5] Brendan Jennings, Sven van der Meer , Sasitharan Balasubramaniam, Dmitri Botvich, Michael O Foghlu and William Donnelly ," Towards Autonomic Management of Communications Networks “, IEEE Communications Magazine, Vol. 45 , No. 10, pp. 112-121, October 2007.

[6] Mauro Femminella , Roberto Francescangeli , Giancula Reali, Jae Woo Lee and Henning Schulzrinne, " $A n$ Enabling Platform for Autonomic Management of the Future Internet ", IEEE Network, vol. 25, no. 6, pp. 24-32 , November 2011.

[7] Xuan Liu, Parikshit Juluri and Deep Medhi ," $A n$ Experimental Study on Dynamic Network Reconfiguration in A Virtualized Network Environment Using Autonomic Management", IEEE/ IFIP International Symposium on Integrated Network Management (ICM2013): MiniConference, pp. 616-622, 2013.

[8] Autonomic Computing, White Paper, "An Architectural blueprint for autonomic computing “, IBM, Third Edition, June 2005.
[9] Ramkumar Cherukuri , Xuan Liu , Andy Bavier, James P.G. Sterbenz and Deep Medhi, “Network Virtualization in GpENI: Framework, Implementation and Integration Experience “, Proceedings of $3^{\text {rd }}$ IEEE/IFIP International Workshop on Management of the Future Internet ( ManFI) , Dublin , Ireland , May $27,2011$.

[10] James P.G. Sterbenz, Deep Medhi , Byrav Ramamurthy, Caterina Scoglio, David Hutchison, Bernhard Plattner, Tricha Anjali, Andrew Scoot, Cort Buffington, Gregory E. Monaco, Don Gruenbacher, Rick McMullen, Justin P. Rohrer, John Sherrell, Pragatheeswaran Angu, Ramkumar Cherukuri , Haiyang Qian and Nidhi Tare ," The Great Plains Environment for Network Innovation ( GpENI): A programmable Testbed for Future Internet Research ", In Proceeding of $6^{\text {th }}$ International Conference on Testbeds and Research Infrastructure for the Development of Networks \& Communities ( Trident Com) Berlin, Germany , May 2010, pp. 428-441.

[11] Thomas Gamer, Marcus Scholler and Roland Bless , “ $A n$ Extensible System for Network Anomaly Detection”, IFIP International Federation for Information Processing 2006, LCNS 4195, pp. 97-108, 2006.

[12] Badis Tebbani and Issam Aib , " GXLA a Language for the Specification of Service Level Agreements ", IFIP International Federation for Information Processing 2006, LCNS 4195, pp. 201-214, 2006.

[13] J. Elias , F. Martignon and A. Capone , “ An Efficient Dynamic Bandwidth Allocation Algorithm for Quality of Service Network*”, IFIP International Federations for Information Processing 2006, LCNS 4195 , pp. 132-145, 2006.

[14] Juan J. Alcaraz, Mario Lopez Martinez, Javier Vales Alonso and Joan Garcia Haro ," Bandwidth Reservation as a Coexistence Strategy in Oppurtinistic Spectrum Access Environments “, IEEE Journal On Selected Areas in Communications, Vol. 32, No. 3, March 2014.

[15] Geeof Huston, Telstra , “Interconnections, Peering and Settlements - Part 1", The Internet Protocol Journal ,Vol. 2 , No.1, March 1999, Available Online : http://www.cisco.com/web/about/ac123/ac147/archived_iss ues/ipj_2-1/peering_and_settlements.html 